\title{
Rates of Reporting Suicidal Ideation and Symptoms of Depression on Children's Depression Inventory in a Paediatric Neurology Sample
}

\author{
Anya Mazur-Mosiewicz ${ }^{1,2}$ • Helen L. Carlson ${ }^{1,3}$ • Cailey Hartwick ${ }^{1}$. \\ Christianne Laliberte $^{1}$ - Emily Tam ${ }^{1}$ • Elisabeth M. S. Sherman ${ }^{1,4,5}$. \\ Brian L. Brooks ${ }^{1,3,4}$
}

Received: 16 October 2014 / Revised: 25 February 2015 / Accepted: 11 March 2015 /Published online: 14 April 2015

(C) American Academy of Pediatric Neuropsychology 2015

\begin{abstract}
Background and Objectives The Children's Depression Inventory (CDI) is frequently used to screen for the symptoms of depression and suicidal thinking during psychological or neuropsychological evaluations. This includes assessment of children with neurological conditions who are at risk of experiencing suicidal thoughts due to general cognitive, psychiatric, and neurological deficits. The purpose of this study was to examine the prevalence and correlates of suicidal thinking and symptoms of depression in youth with neurological disorders, as measured by the CDI. We expected that reporting suicidal ideations would most often occur in children with epilepsy and individuals with low IQ, and positively correlate with impulsivity.

Methods Participants included 313 paediatric neurology patients (mean age $=13.1$ years, $\mathrm{SD}=3.1$, range $=7-17$ ) who underwent neuropsychological assessments, including completion of the CDI.

Results Clinically elevated levels of symptoms of depression were found in $10 \%$ of children, with $18.8 \%$ of the total
\end{abstract}

Anya Mazur-Mosiewicz

amazur-Mosiewicz@thechicagoschool.edu

1 Neurosciences Program, Alberta Children's Hospital, Calgary, Alberta, Canada

2 Clinical Psychology Doctoral Program, Chicago School of Professional Psychology, Chicago, IL, USA

3 Alberta Children's Hospital Research Institute (ACHRI), Calgary, AB, Canada

4 University of Calgary, Calgary, AB, Canada

5 Copeman Healthcare, Calgary, AB, Canada sample endorsing suicidal ideation on the CDI. Suicidal ideation was most commonly endorsed by youth with epilepsy (22.8\%), children between ages 7 and 10 years $(25.8 \%)$, youth with intellectual disabilities ( $40 \%$ for IQ below the 2nd and $70 \%$ for IQ below 0.2 nd percentiles), and girls with attention problems (67\%). Depressive symptoms were significantly correlated with IQ, processing speed, executive functions, attention, parent-reported internalizing behaviours, and gender. Suicidal ideations were best predicted by low verbal intelligence, executive dysfunction, being female, and problems with inattention.

Conclusions Assessments of youth with neurological issues should include a psychological measure, particularly for patients with epilepsy and cognitive disabilities, even at a relatively early age.

Keywords Children's Depression Inventory · Cognition Executive functions $\cdot$ TBI $\cdot$ Epilepsy $\cdot$ Stroke

$\begin{array}{ll}\text { Abbreviations } \\ \text { SB } & \text { Suicidal behaviours } \\ \text { SI } & \text { Suicidal ideation } \\ \text { STI } & \text { Suicidal ideation with intent } \\ \text { ST } & \text { Suicidal thinking without reported intent }\end{array}$

\section{Introduction}

Depression and suicidal ideation assessment is a standard practice in a typical neuropsychological assessment. The measures often used for this purpose are the Children's Depression Inventory (CDI; Kovacs 1992) and Children's Depression Inventory, Second Edition (CDI-2; Kovacs 2011). Although 
the measures are considered to be well-established in a variety of clinical and non-clinical settings (Sattler and Hoge 2006), little is known about the rates of endorsing suicidal thoughts and/or elevated symptoms of depression on the CDI/CDI-2 by children who suffer from serious neurological conditions.

Approximately one third of children report thinking of suicide at some point of their life and $9.7 \%$ of adolescents admit to a suicide attempt (Evans et al. 2005). The prevalence of reporting suicidal intentions in the most recent 6 months ranges between 4 and $34 \%$ (mean of $17 \%$ ), and the prevalence of suicide attempts ranges from 1 to $17 \%$ (mean of $7 \%$ ) (Evans et al. 2005). Variability in these rates is related not only to sociocultural, demographic, cognitive, and other factors but also to the methods used in assessing suicidal intent. Moreover, literature concerning suicidal trends typically highlights that population-specific rates may not apply to other populations of interest (Kokkevi et al. 2012).

Children with neurological conditions may be at particular risk of displaying psychological problems due to two factors: (1) neurophysiological changes in the CNS that are secondary to neurological disease and (2) psychosocial stressors related to the medical condition. These two factors belong to the biopsychosocial model of psychopathology. According to its framework, individuals with neurological issues may display more psychological problems than healthy individuals, regardless of the aetiology of the neurological illness (Lezak et al. 2012; Schoenberg and Scott 2011). Although emotional disturbance has been noted in respect to many areas of the CNS, the brain areas that are typically implicated in the development of cognitive and emotional problems involve the orbital medial frontal and dorsolateral frontal lobe networks. Impairments within these networks may result from a wide range of symptoms such as disinhibition, poor social and emotional control, impulsivity and inappropriate sexual and social behaviours (more related to the orbital medial frontal lobe syndrome) as well as abulia, apathy, emotional restriction, motivation, and lack of initiative (more related to the dorsolateral frontal networks). According to the model, the psychosocial component is related to the process of adjustment to the medical condition, impact of the condition on independence and quality of life, social interactions, family interactions, development of maladaptive coping strategies, functional independence, and other factors that affect both the patient and their environment. Both factors of the biopsychosocial model interact with each other to produce psychological symptoms.

Indeed, physical well-being and the presence of neurological conditions are found to significantly affect suicidal intent and behaviour (Bridge et al. 2006; Kanner 2009b; Simpson and Tate 2007). This primarily involves epilepsy, although other conditions such as traumatic brain injury (TBI) are also known for having high rates of suicidal thoughts and behaviours (Kanner 2009a, b; Kerr et al. 2011; Simpson and Tate 2007). When compared to healthy controls, epilepsy patients are at a threefold higher risk of suicide as a cause of death (Christensen et al. 2007) and at a twofold higher risk of suicidal ideation (Nilsson et al. 2002). For TBI, rates of suicidal intent appear to be positively correlated with the severity of neurological impairment and post-injury psychological distress (Simpson and Tate 2007). These rates appear to be further affected by factors such as psychosis, depression, substance abuse, and weaker cognitive functioning (Simpson and Tate 2007).

Suicidal ideations are frequently reported to relate to cognitive factors including problem solving (Marzuk et al. 2005), impulsivity (Horesh et al. 1999; Hull-Blanks et al. 2004), and overall IQ (Alati et al. 2009). Additionally, girls report higher levels of suicidal intentions and behaviours than boys, but boys display higher rates of suicide completion (Evans et al. 2005); furthermore, the rates of suicidal intentions and behaviours increase as children advance into adolescence (Beautrais 2002; Brent et al. 1999; Groholt et al. 1998; Klonsky and May 2010; Shaffer et al. 1996).

To date, multiple studies have used various reporting measures to examine the correlates and potential predictors of suicidal intention reporting in adults with neurological conditions or neurologically healthy children (Alati et al. 2009; Apter et al. 2003; Beautrais et al. 1998, 1999a, b). However, few studies have focused on the rates of suicidal intentions and/or elevated rates of depression as reported on the $\mathrm{CDI} / \mathrm{CDI}-2$ in children with serious neurological conditions. Furthermore, few studies have compared the rates of reported suicidal thoughts and/or elevated symptoms of depression among different neurological groups (Brent 1986; Camfield et al. 2002).

This study aims to report the rates of endorsing suicidal intent on the CDI/CDI-2 for a sample of children with specific neurological disorders and to identify specific neuropsychological and demographic correlates. We expected that children with epilepsy would report the highest prevalence of suicidal intentions as compared to other populations. We also predicted higher rates of suicidal ideation in children with lower intellectual functioning and higher impulsivity. Thus, we sought to enhance direct patient care by elucidating the psychological presentation and needs of paediatric patients who present to tertiary care neurology clinics.

\section{Materials and Methods}

\section{Participants and Procedure}

Data was extracted from an anonymized neuropsychological database that included consecutively referred patients who underwent an assessment between February 2006 and November 2012 at a tertiary care hospital, Alberta Children's Hospital in Calgary, Alberta, Canada. Participants were included if they had a neurological diagnosis (as determined by the treating neurologist, neurosurgeon, or 
paediatrician), completed a neuropsychological assessment, and had answered either the CDI (Kovacs 1992) or CDI-2 (Kovacs 2011). If a patient was assessed several times, only the initial assessment was used. This project was granted ethical approval by the University of Calgary Research Ethics Board (REB 13-0792) and informed consent was acquired from parents/guardians.

\section{Measures}

The CDI and CDI-2 (Kovacs 1992, 2011) are two consecutive editions of a self-report measure designed to assess the presence of symptoms associated with depression in individuals 7-17 years of age. The CDI and CDI-2 contain 27 and 28 items, respectively. All items included three statements, and respondents were asked to select the statement that best described their feelings. The total raw scores were converted to age- and genderadjusted $T$ scores. Overall $T$ scores of 65 or higher were considered clinically elevated (Kovacs 2003).

$\mathrm{CDI} / \mathrm{CDI}-2$ contains one item that addresses the level of suicidal thinking/intent, and includes (1) "I do not think about killing myself"; (2) "I think about killing myself but I would not do it"; and (3) "I want to kill myself". For the purpose of the current analysis, endorsement of option 2 or 3 was coded as overall suicidal ideation. Information regarding the inventories, test reliability, and validity can be found in their respective manuals (Kovacs 1992, 2011).

Participants were also administered measures of neurocognitive functioning, including tests of IQ, processing speed, memory, attention, executive functions, adaptive functioning, and externalizing and internalizing behaviours (for the list of included measures, see Table 1). Note that overall IQ score was used only to report the rates of endorsing suicidal ideation and depressive symptoms among children with specific ranges of cognitive functioning. In subsequent statistical calculations, including correlational as well as classification analysis, non-verbal intelligence and verbal intelligence scores were used instead to gain greater specificity in understanding the relationship between specific neurocognitive variables and endorsing the symptoms of suicidal thinking and depression on the CDI/CDI-2 .

\section{Analyses}

Initial statistics were completed using the Statistical Package for the Social Sciences (SPSS, IBM Corporation 2010). Kolmogorov-Smirnov tests were performed on overall CDI/ CDI-2 $T$ scores and all other neurocognitive measures to explore distribution normality. Subsequently, Spearman's rho rank correlation coefficients for non-normally distributed data were conducted between overall CDI/CDI-2 $T$ score and selected neurocognitive and demographic variables. A Mann-Whitney $U$ test was performed to explore significant differences in the CDI/CDI- $2 T$ scores across gender. Variables identified as significantly related to overall CDI/CDI-2 $T$ score based on Spearman's rho (at the $p<0.05$ level) were utilized in a subsequent classification and regression tree analysis (CART; Breiman et al. 1984; Lemon et al. 2003).

The CART analysis was chosen over logistic regression or discriminant analysis because it is appropriate for non-normal distributions, is considered robust, is able to handle both categorical and continuous variables (Strobl et al. 2008) and missing data (Therneau and Atkinson 2014). The CART analysis incorporates nonlinear effects without requiring additional variables or variable transformations; outliers have limited impact on results, and there is no theoretical constraint concerning the number and type of variables to be considered as potential explanatory variables (Breiman et al. 1984). A CART analysis is therefore particularly useful when there are few a priori expectations as to the relationships between variables. By contrast, logistic regression and discriminant analysis are sensitive to nonnormal distributions, outliers, and missing data. Through complete case analysis, both typically exclude participants that do not have a data point for every predictor variable, a limitation that CART does not share.

A CART analysis was conducted using the statistical package R (Core Team 2012) to recursively partition groups of variables into progressively more homogenous subgroups by defining thresholds within the predictor variables. The dependent variable used was the absence versus presence of suicidal ideation. Absence of suicidal ideation was defined as selecting option 1 on the CDI: "I do not think about killing myself". Presence of suicidal ideation was defined as either selecting option 2: "I think about killing myself but I would not do it" $O R$ selecting option 3: "I want to kill myself". Seven demographic and neuropsychological variables were assigned as the explanatory variables (gender, verbal intelligence, non-verbal intelligence, processing speed, attention, internalizing behaviours, and executive functioning). Selected explanatory variables were independent so as to avoid violating redundancy assumptions, i.e. overall IQ was not included as an explanatory variable since verbal and non-verbal intelligence were included.

CART determines which variable maximizes the homogeneity (and minimizes the heterogeneity) within a given node for the dependent variable. Subsequent splits increase the model's ability to accurately classify data. In the current study, the CART algorithm determined which variable best differentiated between reporting suicidal ideation or not. This variable was then set aside as 
Table 1 Measurements of neurocognitive functioning

\begin{tabular}{|c|c|}
\hline Cognitive domain & Measure used \\
\hline Intellectual abilities & $\begin{array}{l}\text { Full Scale IQ from } \\
\text { WPPSI-III (Wechsler 2004) } \\
\text { WISC-IV (Wechsler 2003) } \\
\text { WAIS-III/IV (Wechsler 1997a, 2008) }\end{array}$ \\
\hline Processing speed & $\begin{array}{l}\text { Processing Speed Index from } \\
\text { WPPSI-III (Wechsler 2004) } \\
\text { WISC-IV (Wechsler 2003) } \\
\text { WAIS-III/IV (Wechsler 1997a, 2008) }\end{array}$ \\
\hline Verbal intelligence & $\begin{array}{l}\text { Verbal Comprehension Index from } \\
\text { WPPSI-III (Wechsler 2004) } \\
\text { WISC-IV (Wechsler 2003) } \\
\text { WAIS-III/IV (Wechsler 1997a, 2008) }\end{array}$ \\
\hline Non-verbal intelligence & $\begin{array}{l}\text { Perceptual Reasoning Index from } \\
\text { WPPSI-III (Wechsler 2004) } \\
\text { WISC-IV (Wechsler 2003) } \\
\text { WAIS-III/IV (Wechsler 1997a, 2008) }\end{array}$ \\
\hline Recall memory & $\begin{array}{l}\text { For words, Long Delay Free Recall from } \\
\text { CVLT-C (Delis et al. 1994) } \\
\text { CVLT-II (Delis et al. 2000) } \\
\text { For faces, } \\
\text { Faces Delayed from CMS (Cohen 1997) } \\
\text { Faces II from WMS-III (Wechsler 1997b) }\end{array}$ \\
\hline Attention skills & ADHD Rating Scale-IV (DuPaul et al. 1998) \\
\hline Executive functions & Global Executive Composite (GEC) from BRIEF (Gioia et al. 2000) \\
\hline Adaptive functioning & Adaptive Functioning Index from BASC 2 (Reynolds and Kamphus 2004) \\
\hline Externalizing behaviours & Externalizing Behaviors Index from BASC 2 (Reynolds and Kamphus 2004) \\
\hline Internalizing behaviours & Internalizing Behaviours Index from BASC 2 (Reynolds and Kamphus 2004) \\
\hline Inhibition & Inhibition Total Errors from NEPSY, Second Edition (Korkman et al. 2007) \\
\hline Cognitive flexibility & Cognitive Flexibility Index from CNS Vital Signs (Moresville, NC) \\
\hline
\end{tabular}

a terminal node and the differentiation of the remaining variables was again examined. This iterative process resulted in a tree structure consisting of branches that illustrate the relative contributions of each variable in explaining the likelihood of reporting suicidal ideation. To avoid over-fitting, the analysis was automatically terminated when the addition of any more variables did not add any more significantly explanatory nodes (as determined by a plot of relative error and number of splits). No further pruning methods were used. The nodes and the nature of the splits in the resulting tree structure were examined to interpret which variables could most strongly explain the likelihood of reporting suicidal ideation.

To augment the CART, a random forest (RF) analysis (Breiman 2001) was performed. The RF analysis is an iterative bootstrapping procedure that repeatedly performs classification analysis with data subsamples until it converges on a list of explanatory variables. One thousand trees were grown and the mean decrease in Gini (a measure of node impurity) was used as a quantifier for the role a predictor plays in partitioning the data into the defined classes (i.e. higher values indicate a more predictive role in the model).

\section{Results}

Details of demographic characteristics of the sample are presented in Table 2. Performance on neurocognitive measures, CDI/CDI-2 scores, and rates of suicidal ideation (SI) and suicidal thinking with intent (STWI) are reported in Tables 3 and 4.

Correlations between CDI/CDI-2 overall scores, demographic, and neuropsychological variables are presented in Table 5. CDI/CDI-2 overall scores were significantly 
Table 2 Summary demographics of our paediatric neurology patient sample

\begin{tabular}{lr}
\hline Demographics & \\
\hline Age (years), mean \pm SD (range) & $13.0 \pm 3.1(7.0-17.9)$ \\
Duration of the medical condition, mean \pm SD (range) & $5.8 \pm 4.5(0-17.65)$ \\
Parent education (years), mean \pm SD (range) & \\
-Mother & $13.7 \pm 2.6(0-20)$ \\
-Father & $13.6 \pm 3.1(0-20)$ \\
Sex (\%) & \\
-Male & 50.2 \\
-Female & 49.8 \\
Ethnicity (\%) & \\
-Caucasian & 60.1 \\
-Asian & 3.8 \\
-Middle Eastern & 3.2 \\
-Mixed & 3.2 \\
-Hispanic & 2.2 \\
-African & 1.3 \\
-First nations & 1.0 \\
-Other & 2.9 \\
-Not reported & 22.4 \\
Diagnosis (\%) & \\
-Epilepsy & 53.4 \\
-TBI & 17.3 \\
-General neurology & \\
-Stroke & 5.3 \\
-Hydrocephalus & \\
\hline & \\
\hline
\end{tabular}

correlated with verbal intelligence, non-verbal intelligence, processing speed, attention, parent-rated internalizing behaviours, parent-rated externalizing behaviours, and executive function. In addition, gender differences in endorsing depressive symptoms were identified as significant $(n=299$, $p<0.01$ ), with females reporting more symptoms of depression than males. These seven variables were used in the subsequent CART analysis, an illustration of which is displayed in Fig. 1. Five of the seven variables were the most important in explaining the likelihood of suicidal ideation in our sample.

In the CART analysis, verbal intelligence was the most explanatory variable indicating that $70 \%$ of the likelihood of reporting suicidal ideation was explained by a child having a verbal intelligence standard score less than 57.5. Of those children with a verbal intelligence standard score above 57.5, executive dysfunction (i.e., scores on the BRIEF-P higher than $T=50.5$ ) was predictive of $20.5 \%$ of reports of suicidal ideation. The third explanatory variable was gender and indicated that females had a higher likelihood of reporting SI $(28.1 \%)$ compared to males $(13.6 \%)$. The final factor in explaining the likelihood of SI was parent rating of attention, indicating that $66.7 \%$ of children endorsing suicidal ideation were rated higher than the 98.5 th percentile on the attention rating scale.

RF analysis confirmed the CART results, ranking verbal intelligence (mean decrease in Gini index $(\mathrm{MDG})=13.17)$, executive functioning $(\mathrm{MDG}=11.88)$, and attention $(\mathrm{MDG}=$ 11.76) as the top three variables of importance when classifying predictors of suicidal ideation. Of note, non-verbal intelligence $(\mathrm{MDG}=10.5)$ and processing speed $(\mathrm{MDG}=10.44)$ were also identified as predictors.

\section{Discussion}

The current study provides rates and correlates of suicidal ideation reported by children and adolescents on the CDI/ CDI-2 seen in a large Canadian tertiary neurological centre. For our sample, the overall rate of reporting recent suicidal ideation was $18.8 \%$. This falls within the population estimate of 16 to $26 \%$, which is the $95 \%$ confidence interval of reporting suicidal thoughts of any kind in a large international review of suicidal trends in children (Evans et al. 2005). Although in general, rates of suicidal ideation reported among children with neurological conditions on the CDI/CDI-2 are similar to these observed in other paediatric groups, a closer look at the population breakdown suggests that these rates differ by the type of neurological diagnosis, overall IQ level, and age. As hypothesized, the highest rates of suicidal ideation were reported by children with extremely low overall IQ, although it is questionable whether these endorsements 
Table 3 Rates of endorsing suicidal ideation and depressive symptoms

\begin{tabular}{|c|c|c|c|c|c|c|c|}
\hline CDI performance & Number & $\begin{array}{l}\text { Mean total } \\
T \text { score }\end{array}$ & $\mathrm{SD}$ & Range & $\begin{array}{l}\% \text { clinically } \\
\text { elevated }\end{array}$ & $\begin{array}{l}\% \text { endorsing suicidal } \\
\text { ideation }(\mathrm{SI})\end{array}$ & $\begin{array}{l}\% \text { endorsing suicidal } \\
\text { thinking with intent } \\
\text { (STWI) }\end{array}$ \\
\hline CDI in overall sample & 313 & 49.9 & 11.3 & $34-89$ & 10.2 & 18.8 & 1.9 \\
\hline \multicolumn{8}{|l|}{ CDI across diagnostic groups } \\
\hline Epilepsy patients & 167 & 50.9 & 11.6 & $35-89$ & 12.0 & 22.8 & 3.0 \\
\hline TBI patients & 54 & 50.6 & 11.5 & $34-87$ & 11.1 & 11.1 & 1.9 \\
\hline General neurology patients & 48 & 48.1 & 12.3 & $36-81$ & 10.4 & 16.7 & 0.0 \\
\hline Stroke patients & 28 & 48.0 & 8.4 & $37-71$ & 3.6 & 17.9 & 0.0 \\
\hline Hydrocephalus patients & 16 & 46.2 & 5.9 & $37-56$ & 0.0 & 12.5 & 0.0 \\
\hline \multicolumn{8}{|l|}{ CDI across intellectual groups } \\
\hline IQ below 2nd percentile & 40 & 54.8 & 12.7 & $37-83$ & 20.0 & 40.0 & 7.5 \\
\hline IQ between 2 nd and 15 th percentile & 65 & 50.4 & 11.0 & $36-89$ & 9.2 & 23.1 & 1.5 \\
\hline IQ at 16 th percentile or above & 109 & 48.9 & 10.4 & $34-87$ & 9.2 & 14.7 & 0.0 \\
\hline \multicolumn{8}{|l|}{ CDI across age groups } \\
\hline Age 7 to less than 10 & 66 & 52.3 & 11.3 & $37-88$ & 10.6 & 25.8 & 3.0 \\
\hline Age 10 to less than 13 & 87 & 49.0 & 10.9 & $37-82$ & 8.0 & 24.1 & 3.4 \\
\hline Age 13 to less than 16 & 93 & 48.1 & 10.6 & $34-89$ & 7.5 & 11.8 & 1.1 \\
\hline Age 16 to less than 18 & 67 & 51.3 & 13.1 & $36-89$ & 16.4 & 14.9 & 0.0 \\
\hline
\end{tabular}

To be considered clinically elevated, CDI total $T$ score had to be 65 or above

$C D I$ Children's Depression Inventory, $S D$ standard deviation, $\%$ percent

reflected the actual suicidal intent and/or were made in full understanding of what suicide is.

Our subsequent, more specific classification analysis indicated that $70 \%$ of children with extremely low verbal IQ endorsed some level of suicidal ideation on the measure. Of the remaining sample, the most at risk were girls with significant attention difficulties, with approximately $67 \%$ of these children reporting recent suicidal ideation. Our findings indicate that significant correlates of elevated symptoms of depression are gender, verbal and non-verbal IQ, executive and attention difficulties, and internalizing behaviours. Surprisingly, age and age of onset were not identified in our study as significant correlates of elevated symptoms of depression.

\section{Type of Neurological Diagnosis}

With respect to neurological diagnoses, we hypothesized that children with epilepsy would endorse the highest level of suicidal ideation as compared to other groups. Indeed, almost $23 \%$ of children diagnosed with epilepsy reported thinking of suicide, including $3 \%$ of the sample reporting intent for suicide, suggesting that the incidence of suicidal ideation in these children is strikingly higher than among other neurological populations, such as children with TBI or hydrocephalus. This appears to be supported by the literature that provides evidence of children with epilepsy displaying suicidal ideation more often than any other medical group (Nilsson et al. 2002) as well as active suicidal behaviours (Brent 1986) (Christensen et al. 2007). In our study, children with epilepsy also endorsed symptoms of depression at the highest rate when compared to other neurological groups, supporting the notion that epilepsy is related to symptoms of depression (Camfield et al. 2002). This finding is of particular concern as paediatric epilepsy and depression are considered risk factors for suicide later in life. For example, a Swedish cohort study suggested that patients with symptoms of depression who were diagnosed with epilepsy in adolescence were at the highest risk of suicide, particularly in light of limited neurological follow-up (Camfield et al. 2002).

Children with early epilepsy onset and who display a longstanding history of seizures are at a higher risk of cognitive problems, particularly children with difficult-to-control seizures (Baker et al. 2011; Sarkis et al. 2013). This could partially explain why the current study observes the highest rates of suicidal ideation in this population. It is possible that not only epilepsy as a disorder but also its secondary effects on cognition contribute to the observed differences in suicidal ideation among neurological groups. Taken together, these findings illustrate the necessity of preventive programmes, early screening, and access to psychological interventions for these children and highlight the necessity of including psychological services as integral parts of paediatric epilepsy teams.

\section{Intellectual Functioning}

Children with overall IQ impairment were at the highest risk of endorsing suicidal ideation, which is supportive of research 
Table 4 Cognitive abilities in paediatric neurology patients administered with the CDI

\begin{tabular}{|c|c|c|c|c|c|}
\hline Cognitive domains & Number & Mean & SD & Range & $\%$ impaired \\
\hline Overall intelligence (index score) & 214 & 83.9 & 17.5 & $40-128$ & 20.6 \\
\hline Verbal intelligence (index score) & 190 & 84.7 & 16.1 & $45-128$ & 20.5 \\
\hline Non-verbal intelligence (index score) & 197 & 87.0 & 18.1 & $45-138$ & 18.3 \\
\hline Processing speed (index score) & 282 & 83.2 & 15.9 & $42-135$ & 21.6 \\
\hline Verbal memory, word list ( $z$ score) & 261 & -0.5 & 1.2 & $-4.0-2.0$ & 24.1 \\
\hline Visual memory, faces (scaled score) & 193 & 8.5 & 3.6 & $1-17$ & 15.0 \\
\hline Inhibition (scaled score) & 151 & 7.2 & 4.0 & $1-15$ & 29.8 \\
\hline Cognitive flexibility (index score) & 126 & 89.0 & 18.8 & $29-133$ & 15.1 \\
\hline Attention, parent rating (percentile) ${ }^{\mathrm{a}}$ & 278 & 71.1 & 25.6 & $5-99$ & 11.9 \\
\hline Externalizing behaviours, parent ratings $(t \text { score })^{\mathrm{a}}$ & 65 & 54.4 & 14.0 & $36-95$ & 13.8 \\
\hline Internalizing behaviours, parent ratings $(t \text { score })^{\mathrm{a}}$ & 65 & 57.3 & 13.9 & $34-88$ & 16.9 \\
\hline Executive functioning, parent rating $(t \text { score })^{\mathrm{a}}$ & 279 & 60.0 & 11.9 & 34-92 & 23.3 \\
\hline Adaptive functioning, parent rating $(t \text { score })^{\mathrm{a}}$ & 65 & 44.8 & 11.0 & $21-67$ & 10.8 \\
\hline
\end{tabular}

Impaired is defined as being at or below the 2nd percentile (e.g. 2 standard deviations below the mean). Index scores have a mean $=100$ and $\mathrm{SD}=15$. Percentile scores have a mean $=50$ and range from 1 to $99 . T$ scores have a mean $=50$ and $\mathrm{SD}=10 . Z$ scores have a mean $=0$ and $\mathrm{SD}=1$. Scaled scores have a mean $=10$ and $\mathrm{SD}=3$.

CDI Children's Depression Inventory

${ }^{a}$ Higher scores are reflective of lower functioning (or more problems)

showing that lower cognitive abilities are significantly related to reporting suicide-related thoughts and depression (Alati et al. 2009; Fergusson et al. 2005a, b; Gunnell et al. 2005). Previous studies clearly show that children with lower IQs are at higher risk of thinking about suicide regardless of the assessment mode (Alati et al. 2009) and suicidal behaviours (Alati et al. 2009; Fergusson et al. 1999; Gunnell et al. 2005; O'Toole and Cantor 1995), particularly in the presence of childhood conduct problems and complicated family social circumstances (Diaconu and Turecki 2009; Fergusson et al. 2005a, b). Our additional finding that verbal and non-verbal IQ are significant predictors of suicidal ideation adds more specificity to such literature. Subsequent studies could further elucidate the relative contributions of each facet of IQ in this population.

Although it should be acknowledged that children with impaired intellectual functioning may either have some difficulty understanding the content of the CDI/CDI-2 questions or
Table 5 Correlations between cognitive domains and overall score of CDI for paediatric neurology patients

\begin{tabular}{|c|c|c|}
\hline Demographic and cognitive domains & Number & $\begin{array}{l}\text { Overall CDI score } \\
\text { Spearman's rho }\end{array}$ \\
\hline Age (years) & 313 & -0.074 \\
\hline Duration of the medical condition & 186 & -0.061 \\
\hline Verbal intelligence (index score) & 190 & $-0.170 * *$ \\
\hline Non-verbal intelligence (index score) & 197 & $-0.207^{* *}$ \\
\hline Processing speed (index score) & 282 & $-0.118^{*}$ \\
\hline Verbal memory, word list ( $z$ score) & 261 & -0.075 \\
\hline Visual memory, faces (scaled score) & 193 & -0.020 \\
\hline Inhibition (scaled score) & 151 & -0.029 \\
\hline Cognitive flexibility (index score) & 126 & -0.103 \\
\hline Attention, parent rating (percentile) ${ }^{\mathrm{a}}$ & 278 & $0.211^{* *}$ \\
\hline Externalizing behaviours, parent ratings $(t \text { score })^{\mathrm{a}}$ & 65 & 0.229 \\
\hline Internalizing behaviours, parent ratings $(t \text { score })^{\mathrm{a}}$ & 65 & $0.403^{* *}$ \\
\hline Executive functioning, parent rating $(t \text { score })^{\mathrm{a}}$ & 279 & $0.214^{* *}$ \\
\hline Adaptive functioning, parent rating $(t \text { score })^{\mathrm{a}}$ & 65 & -0.174 \\
\hline
\end{tabular}

CDI Children's Depression Inventory

${ }^{*} p<.05 ; * * p<.01$

${ }^{a}$ Higher scores represent more problems 


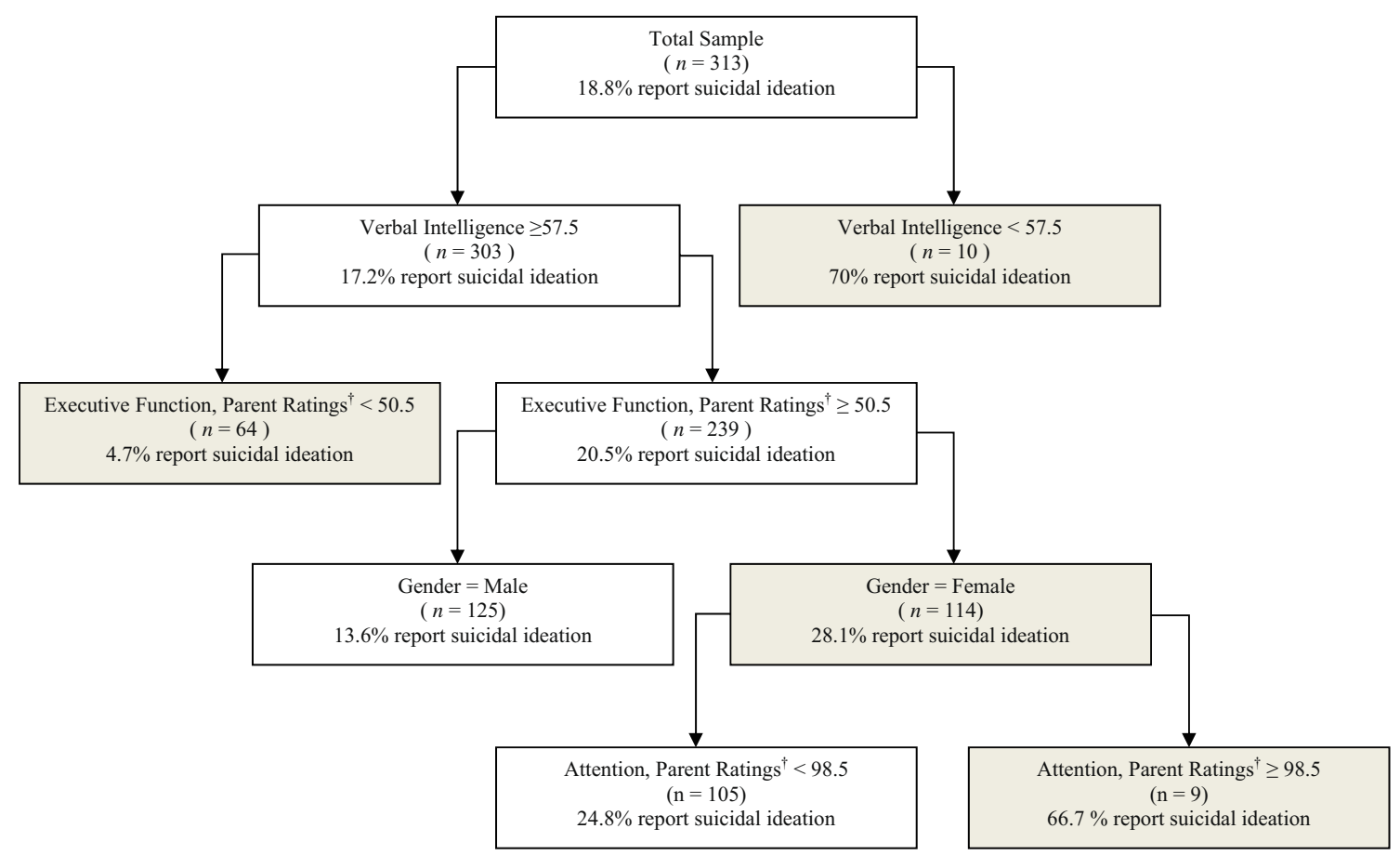

Fig. 1 A simplified classification tree for reported suicidal ideation. SI suicidal ideations. Dagger indicates that higher scores are reflective of lower functioning (or more problems). Shaded nodes indicate nodes of interest

not fully appreciate the meaning of suicide, it does not necessarily mean that these children are not capable of thinking about suicide or engaging in suicidal behaviours. Research shows that this population is vulnerable to developing suicide-related thoughts and engaging in suicidal behaviours, particularly children with severe/profound intellectual deficits (Hardan and Sahl 1999). Previous research shows that approximately $22 \%$ of children with an IQ between 50 and 70 reported thinking about suicide (Pack et al. 1998), and half of the children admitted to inpatient psychiatric units with a dual diagnosis of intellectual disability and psychiatric disorders (ages $6-17 ; 16 \%$ having severe or profound impairment) displayed concerning levels of suicide-related thoughts and/ or suicidal behaviours (Johnson et al. 1995). Rates reported by these studies do not necessary apply to the current population of interest; nevertheless, these studies highlight that regardless of a type of neurological diagnosis, children with intellectual disabilities, including profound and severe disabilities, are at risk of reporting suicide-related thoughts and engaging in suicidal acts.

\section{Age}

Depressive symptoms and suicidal ideations did not increase alongside age as suggested by previous research (Beautrais 2002; Brent et al. 1999; Groholt et al. 1998; Klonsky and May 2010; Shaffer et al. 1996). In fact, children between ages 7 and 9 reported more suicidal ideations than the older groups, although age was not significantly correlated with symptoms of depression. Although it is possible that very young children may not be aware of the full meaning behind the concepts of suicide, it is documented that children as young as 7 years old are capable of suicide-related thoughts (Kovacs 2011) and behaviours (Giraud et al. 2013). Within the Canadian population, it has been reported that children as young as 5 years old do attempt suicide (Stewart et al. 2001), suggesting that even if very young children may not have full understanding of the consequences of suicidal behaviour, they do intentionally engage in them. This suggests that any reports of suicide-related thoughts by these children should not be automatically dismissed as invalid. Rather, closer attention should be given to the reasons behind reporting suicidality and full assessment options should be available for these patients.

\section{Correlates of Suicidal Ideation on the CDI/CDI-2 and Symptoms of Depression}

In the current analyses, internalizing behaviours observed by a parent did significantly correlate with patient-reported depressive symptoms, but were not sufficiently explanatory according to the CART analysis. This suggests that parents perceive symptoms of internalizing behaviours, including symptoms of depression; however, parental perception of internalizing behaviours does not effectively identify the groups of children who are at risk of reporting suicidal ideation on the CDI/CDI2. Thus, stand-alone parental ratings of internalizing behaviours may not be a strong predictor of suicidal ideation in youth, and psychological assessments should be conducted 
with children regardless of their parents' perception of their symptoms of mood problems.

The current study highlights the risk of endorsing suicidal ideation in females who display significant attention problems. This finding is not surprising, as girls consistently report higher levels of suicide-related thoughts and behaviours than boys (Evans et al. 2005). Although symptoms of attention problems significantly predict suicidal behaviours (Horesh et al. 1999; Hull-Blanks et al. 2004; Renaud et al. 2008), this finding is typically associated with males, and this study suggests that females with attention issues may also be at risk.

\section{Limitations}

It is thought that children with low IQ display high rates of psychopathology, placing them at higher risk of depression and suicide, particularly if they present with depressive symptoms, past sexual or physical abuse, presence of oppositionaldefiant disorder, recent family loss, sleep problems, social withdrawal, comorbid affective disorders, female gender, and younger age (Hardan and Sahl 1999; Hassiotis et al. 2011; Johnson et al. 1995; Seltzer et al. 2005; Walters et al. 1995). The current project was not able to address all of these factors, and therefore, it is possible that the relatively small correlations observed are attributable to the limited number of variables used. Future projects should strive to create inclusive models by using neurocognitive, social, psychological, and demographic variables as well as additional cognitive domains such as social problem solving or planning abilities. Moreover, past research indicates that thinking about suicide and depression may be moderated by social, family, and economic variables, as well as comorbid psychiatric issues, which was not explored in the current project (Fergusson et al. 2005a, b).

Since this study focused on the CDI/CDI-2, the reported rates of suicidal ideation may not reflect rates of suiciderelated thoughts that are measured via anonymous questionnaires or direct interview. Another serious limitation of this project is a lack of a healthy control comparison group. The current study was conducted using a Canadian tertiary neurology sample and may not reflect rates among tertiary neurological populations in other countries (Evans et al. 2005). Finally, the CART analysis is considered an exploratory technique and does not offer predictability statistics (Dietterich 2000). As such, future studies should investigate whether the current findings can be confirmed with predictive analyses such as RF.

\section{Conclusions}

The prevalence of reporting suicide-related thoughts on the $\mathrm{CDI} / \mathrm{CDI}-2$ in our paediatric neurological population is about
$20 \%$, with $10 \%$ of children reporting clinically elevated symptoms of depression. The results suggest that some personal variables, such as gender, IQ, and attention problems, as well as the presence of specific neurological diagnosis can help predict which children are more likely to report thoughts related to suicide. However, the results also suggest some caution in relying exclusively on parental reports as they may not be effective at identifying children who are at risk. Overall, the results support the notion that children who display neurological impairment should be routinely screened for signs of depression and suicide-related thoughts regardless of age and parental reports.

Compliance with Ethical Statement All procedures followed were in accordance with the ethical standards of the responsible committee on human experimentation (institutional and national) and with the Helsinki Declaration of 1975, as revised in 2000. Informed consent was obtained from all patients and their legal guardians for being included in the study. No animal studies were carried out by the authors for this article.

Funding No funding was secured for this study.

Conflict of Interest Anya Mazur-Mosiewicz, Helen L. Carlson, Cailey Hartwick, Christianne Laliberte, Emily Tam, Elisabeth M.S. Sherman, and Brian L. Brooks declare that they have no conflict of interest.

\section{References}

Alati, R., Gunnell, D., Najman, J., Williams, G., \& Lawlor, D. (2009). Is IQ in childhood associated with suicidal thoughts and attempts? Findings from the Mater University Study of Pregnancy and its outcomes. Suicide and Life-threatening Behavior, 39(3), 282-293. doi:10.1521/suli.2009.39.3.282.

Apter, A., Horesh, N., Gothelf, D., Zalsman, G., Erlich, Z., \& Soreni, N. (2003). Depression and suicidal behavior in adolescent inpatients with obsessive compulsive disorder. Journal of Affective Disorders, 75(2), 181-189.

Baker, G. A., Taylor, J., \& Aldenkamp, A. P. (2011). Newly diagnosed epilepsy: cognitive outcome after 12 months. Epilepsia, 52(6), 1084-1091. doi:10.1111/j.1528-1167.2011.03043.x.

Beautrais, A. L. (2002). Gender issues in youth suicidal behaviour. Emergency Medicine (Fremantle, W.A.), 14(1), 35-42.

Beautrais, A. L., Joyce, P. R., \& Mulder, R. T. (1998). Youth suicide attempts: a social and demographic profile. The Australian and New Zealand Journal of Psychiatry, 32(3), 349-357.

Beautrais, A. L., Joyce, P. R., \& Mulder, R. T. (1999a). Cannabis abuse and serious suicide attempts. Addiction, 94(8), 1155-1164.

Beautrais, A. L., Joyce, P. R., \& Mulder, R. T. (1999b). Personality traits and cognitive styles as risk factors for serious suicide attempts among young people. Suicide and Life-threatening Behavior, 29(1), 37-47.

Breiman, L. (2001). Random forests. Machine Learning, 45(1), 5-32.

Breiman, L., Friedman, J. H., Olshen, R. A., \& Stone, C. J. (1984). Classification and regression trees. New York: Chapman \& Hall/CRC

Brent, D. A. (1986). Overrepresentation of epileptics in a consecutive series of suicide attempters seen at a children's hospital, 19781983. Journal of the American Academy of Child Psychiatry, 25(2), 242-246. 
Brent, D. A., Baugher, M., Bridge, J., Chen, T., \& Chiappetta, L. (1999). Age- and sex-related risk factors for adolescent suicide. Journal of the American Academy of Child and Adolescent Psychiatry, 38(12), 1497-1505. doi:10.1097/00004583-199912000-00010.

Bridge, J. A., Goldstein, T. R., \& Brent, D. A. (2006). Adolescent suicide and suicidal behavior. Journal of Child Psychology and Psychiatry, 47(3-4), 372-394. doi:10.1111/j.1469-7610.2006.01615.x.

Camfield, C. S., Camfield, P. R., \& Veugelers, P. J. (2002). Death in children with epilepsy: a population-based study. Lancet, 359(9321), 1891-1895. doi:10.1016/S0140-6736(02)08779-2.

Christensen, J., Vestergaard, M., Mortensen, P. B., Sidenius, P., \& Agerbo, E. (2007). Epilepsy and risk of suicide: a populationbased case-control study. Lancet Neurology, 6(8), 693-698. doi: 10.1016/S1474-4422(07)70175-8.

Cohen, M. J. (1997). Children's Memory Scale. San Antonio, TX: The Psychological Corporation.

Delis, D. C., Kramer, J. H., Kaplan, E., \& Ober, B. A. (1994). California Verbal Learning Test-Children's Version. San Antonio, TX: Psychological Corporation.

Delis, D. C., Kramer, J. H., Kaplan, E., \& Ober, B. A. (2000). California Verbal Learning Test (2nd ed.). San Antonio, TX: Psychological Corporation.

Diaconu, G., \& Turecki, G. (2009). Family history of suicidal behavior predicts impulsive-aggressive behavior levels in psychiatric outpatients. Journal of Affective Disorders, 113(1-2), 172-178. doi:10. 1016/j.jad.2008.03.028.

Dietterich, T. G. (2000). An experimental comparison of three methods for constructing ensembles of decision trees: bagging, boosting, and randomization. Machine Learning, 40(2), 139-157.

DuPaul, G. J., Power, T. J., Anastopoulos, A. D., \& Reid, R. (1998). ADHD Rating Scale-IV: checklists, norms, and clinical interpretation. New York: Guilford.

Evans, E., Hawton, K., Rodham, K., \& Deeks, J. (2005). The prevalence of suicidal phenomena in adolescents: a systematic review of population-based studies. Suicide and Life-threatening Behavior, 35(3), 239-250.

Fergusson, D. M., Horwood, L. J., \& Beautrais, A. L. (1999). Is sexual orientation related to mental health problems and suicidality in young people? Archives of General Psychiatry, 56(10), 876-880.

Fergusson, D. M., Horwood, L. J., \& Ridder, E. M. (2005a). Show me the child at seven II: childhood intelligence and later outcomes in adolescence and young adulthood. Journal of Child Psychology and Psychiatry, 46(8), 850-858. doi:10.1111/j.1469-7610.2005.01472.x.

Fergusson, D. M., Horwood, L. J., Ridder, E. M., \& Beautrais, A. L. (2005b). Suicidal behaviour in adolescence and subsequent mental health outcomes in young adulthood. Psychological Medicine, 35(7), 983-993.

Gioia, G. A., Isquith, P. K., Guy, S. C., \& Kenworthy, L. (2000). Behavior rating inventory of executive function. Child Neuropsychology, 6(3), 235-238.

Giraud, P., Fortanier, C., Fabre, G., Ghariani, J., Guillermain, Y., \& Rouviere, N. (2013). Suicide attempts by young adolescents: epidemiological characteristics of 51715 -year-old or younger adolescents admitted in French emergency departments. Archives of Pediatrics, 20(6), 608-615. doi:10.1016/j.arcped.2013.03.024.

Groholt, B., Ekeberg, O., Wichstrom, L., \& Haldorsen, T. (1998). Suicide among children and younger and older adolescents in Norway: a comparative study. Journal of the American Academy of Child and Adolescent Psychiatry, 37(5), 473-481. doi:10.1097/00004583199805000-00008.

Gunnell, D., Magnusson, P. K., \& Rasmussen, F. (2005). Low intelligence test scores in 18 year old men and risk of suicide: cohort study. BMJ, 330(7484), 167. doi:10.1136/bmj.38310.473565.8F.

Hardan, A., \& Sahl, R. (1999). Suicidal behavior in children and adolescents with developmental disorders. Research in Developmental Disabilities, 20(4), 287-296.
Hassiotis, A., Tanzarella, M., Bebbington, P., \& Cooper, C. (2011). Prevalence and predictors of suicidal behaviour in a sample of adults with estimated borderline intellectual functioning: results from a population survey. Journal of Affective Disorders, 129(1-3), 380384. doi:10.1016/j.jad.2010.10.002.

Horesh, N., Gothelf, D., Ofek, H., Weizman, T., \& Apter, A. (1999). Impulsivity as a correlate of suicidal behavior in adolescent psychiatric inpatients. Crisis, 20(1), 8-14.

Hull-Blanks, E. E., Kerr, B. A., \& Robinson Kurpius, S. E. (2004). Risk factors of suicidal ideations and attempts in talented, at-risk girls. Suicide and Life-threatening Behavior, 34(3), 267-276. doi:10. 1521/suli.34.3.267.42782.

IBM Corporation. (2010). IBM SPSS Statistics for Windows, Version 19.0. Armonk, NY: IBM Corp.

Johnson, C. R., Handen, B. L., Lubetsky, M. J., \& Sacco, K. A. (1995). Affective disorders in hospitalized children and adolescents with mental retardation: a retrospective study. Research in Developmental Disabilities, 16(3), 221-231.

Kanner, A. M. (2009a). Depression and epilepsy: do glucocorticoids and glutamate explain their relationship? Current Neurology and Neuroscience Reports, 9(4), 307-312.

Kanner, A. M. (2009b). Psychiatric issues in epilepsy: the complex relation of mood, anxiety disorders, and epilepsy. Epilepsy and Behavior, 15(1), 83-87. doi:10.1016/j.yebeh.2009.02.034.

Kerr, M. P., Mensah, S., Besag, F., de Toffol, B., Ettinger, A., \& Kanemoto, K. (2011). International consensus clinical practice statements for the treatment of neuropsychiatric conditions associated with epilepsy. Epilepsia, 52(11), 2133-2138. doi:10.1111/j.15281167.2011.03276.x.

Klonsky, E. D., \& May, A. (2010). Rethinking impulsivity in suicide. Suicide and Life-threatening Behavior, 40(6), 612-619. doi:10. 1521/suli.2010.40.6.612.

Kokkevi, A., Rotsika, V., Arapaki, A., \& Richardson, C. (2012). Adolescents' self-reported suicide attempts, self-harm thoughts and their correlates across 17 European countries. Journal of Child Psychology and Psychiatry, 53(4), 381-389. doi:10.1111/j.14697610.2011.02457.x.

Korkman, M., Kirk, U., \& Kemp, S. (2007). NEPSY (2nd ed.). San Antonio, TX: Harcourt Assessment.

Kovacs, M. (1992). Children's Depression Inventory Manual. New York: Multi-Health Systems.

Kovacs, M. (2003). Children's Depression Inventory Technical Manual Update. Toronto, ON: Multi-Health Systems.

Kovacs, M. (2011). Children's Depression Inventory 2nd Edition Technical Manual. Toronto, ON: Multi-Health Systems Inc.

Lemon, S. C., Roy, J., Clark, M. A., Friedmann, P. D., \& Rakowski, W. (2003). Classification and regression tree analysis in public health: methodological review and comparison with logistic regression. Annals of Behavioral Medicine, 26(3), 172-181.

Lezak, M. D., Howieson, D. B., Bigler, E. D., \& Tranel, D. (2012). Neuropsychological assessment. Oxford: Oxford University Press.

Marzuk, P. M., Hartwell, N., Leon, A. C., \& Portera, L. (2005). Executive functioning in depressed patients with suicidal ideation. Acta Psychiatrica Scandinavica, 112(4), 294-301. doi:10.1111/j.16000447.2005.00585.x.

Nilsson, L., Ahlbom, A., Farahmand, B. Y., Asberg, M., \& Tomson, T. (2002). Risk factors for suicide in epilepsy: a case control study. Epilepsia, 43(6), 644-651.

O’Toole, B. I., \& Cantor, C. (1995). Suicide risk factors among Australian Vietnam era draftees. Suicide and Life-threatening Behavior, 25(4), 475-488.

Pack, R. P., Wallander, J. L., \& Browne, D. (1998). Health risk behaviors of African American adolescents with mild mental retardation: prevalence depends on measurement method. American Journal of Mental Retardation, 102(4), 409-420. 
R Core Team. (2012). R: a language and environment for statistical computing. Vienna, Austria: R Foundation for Statistical Computing.

Renaud, J., Berlim, M. T., McGirr, A., Tousignant, M., \& Turecki, G. (2008). Current psychiatric morbidity, aggression/impulsivity, and personality dimensions in child and adolescent suicide: a case-control study. Journal of Affective Disorders, 105(1-3), 221-228. doi: 10.1016/j.jad.2007.05.013.

Reynolds, C. R., \& Kamphus, R. W. (2004). Behavior assessment system for children (2nd ed.). Circle Pines, MN: AGS Publishing.

Sarkis, R. A., Pietras, A. C., Cheung, A., Baslet, G., \& Dworetzky, B. (2013). Neuropsychological and psychiatric outcomes in poorly controlled idiopathic generalized epilepsy. Epilepsy and Behavior, 28(3), 370-373. doi:10.1016/j.yebeh.2013.05.020.

Sattler, J. M., \& Hoge, R. D. (2006). Assessment of children: behavioral, social, and clinical foundations (5th ed.). San Diego: Jerome M. Sattler Inc.

Schoenberg, M. R., \& Scott, J. G. (Eds.). (2011). The little black book of neuropsychology: a syndrome-based approach. New York: Springer.

Seltzer, M. M., Floyd, F., Greenberg, J., Lounds, J., Lindstromm, M., \& Hong, J. (2005). Life course impacts of mild intellectual deficits. American Journal of Mental Retardation, 110(6), 451-468. doi:10. 1352/0895-8017(2005)110[451:LCIOMI]2.0.CO;2.

Shaffer, D., Gould, M. S., Fisher, P., Trautman, P., Moreau, D., \& Kleinman, M. (1996). Psychiatric diagnosis in child and adolescent suicide. Archives of General Psychiatry, 53(4), 339-348.

Simpson, G., \& Tate, R. (2007). Suicidality in people surviving a traumatic brain injury: prevalence, risk factors and implications for clinical management. Brain Injury, 21(13-14), 1335-1351. doi:10. 1080/02699050701785542.

Stewart, S. E., Manion, I. G., Davidson, S., \& Cloutier, P. (2001). Suicidal children and adolescents with first emergency room presentations: predictors of six-month outcome. Journal of the American Academy of Child and Adolescent Psychiatry, 40(5), 580-587. doi:10.1097/ 00004583-200105000-00018.

Strobl, C., Boulesteix, A. L., Kneib, T., Augustin, T., \& Zeileis, A. (2008). Conditional variable importance for random forests. $B M C$ Bioinformatics, 9, 307. doi:10.1186/1471-2105-9-307.

Therneau, T. M., \& Atkinson, E. J. (2014). An introduction to recursive partitioning using the RPART routines. http://cran.r-project.org/web/ packages/rpart/vignettes/longintro.pdf

Walters, A. S., Barrett, R. P., Knapp, L. G., \& Borden, M. C. (1995). Suicidal behavior in children and adolescents with mental retardation. Research in Developmental Disabilities, 16(2), 85-96.

Wechsler, D. (1997a). Wechsler Adult Intelligence Scale, third edition, administration and scoring manual. San Antonio, TX: The Psychological Corporation.

Wechsler, D. (1997b). Wechsler Memory Scale (3rd ed.). San Antonio, TX: The Psychological Corporation.

Wechsler, D. (2003). Wechsler Intelligence Scale for Children (4th ed.). San Antonio, TX: The Psychological Corporation.

Wechsler, D. (2004). Wechsler Preschool and Primary Scale of Intelligence, third edition, Canadian. San Antonio, TX: The Psychological Corporation.

Wechsler, D. (2008). Wechsler Adult Intelligence Scale (4th ed.). San Antonio, TX: Pearson. 